\title{
Linguistic Fuzzy Rough Sets for Multi Criteria Group Decision Making
}

\author{
Hai Wang and Zeshui $\mathrm{Xu}^{*}$ \\ School of Economics and Management, Southeast University, Nanjing, China \\ *Corresponding author
}

\begin{abstract}
Linguistic variables usually take the form of a base term modified by a hedge in qualitative evaluations of multi criteria decision making problems. In this paper, the linguistic fuzzy rough set is presented to objectively model non-inclusive hedges, such as more or less and roughly, with semantics. Based on the predefined resemblance relation, hedges can be represented by some compact formulae whenever the linguistic term set is uniformed distributed or non-uniformed distributed. Then a corresponding multi criteria group decision making approach is supplied for potential application and illustrated by a problem of evaluating new energy technologies.
\end{abstract}

Keywords-multi criteria group decision making; linguistic fuzzy rough set; linguistic hedges; resemblance relation

\section{INTRODUCTION}

Multi-criteria group decision making (MCGDM) problems which refer to evaluating, prioritizing or selecting among some available alternatives with respect to multi-criteria are very common in practice [1]. To express preference information "accurately" with higher confidence, linguistic terms are commonly used by decision makers (DM) or experts. Thus linguistic representation models, serving as techniques of computing with words (CWW) [2], are vital for MCGDM in linguistic setting. Based on the fuzzy linguistic approach, two classes of linguistic models have been developed, i.e., the semantic model and the symbolic computing model [3].

Generally, a linguistic variable is a variable whose values are words or sentences in a natural or artificial language [4]. A linguistic term set (LTS) is considered to let all the terms distributed on a predefined ordered scale. The semantics of the terms are described by membership functions. The linear trapezoidal membership functions are good enough to capture the vagueness of these linguistic assessments [5]. As seen in Figure 1, the triangular membership functions, as a particular case, are convenient for application. Till now, LTSs with different kinds of distribution are presented, such as uniformly and non-uniformly distributed terms. Two computational models, i.e., the virtual terms model and the 2-tuple model, are presented to avoid the loss of information. To enrich the linguistic representation model, two extended models have been proposed to consider more than one term, which are uncertain linguistic terms and hesitant fuzzy LTSs [6].

However, the existing extensions are still limited. For example, a decision group is authorized to evaluate an energy technology with respect to its contribution to regional development by a LTS. Because of the uncertainties and risks of both the current status and the future, the organization may be not very sure to express the opinion by one certain term. Thus the most natural linguistic expression they used may be "more or less high" if "high" is supported by most of the evidences. But it's hard to handle this kind of linguistic expressions directly. In this content, some alternative solutions have to be used, e.g. considering another LTS with small granularity, representing it by an interval such as [medium, very high] or listing all possible terms in a HFLTS such as \{medium, high, very high\}. From another aspect, once a term is selected, some linguistic hedges may be used to express one's preference more accurately, or to raise the confident level of the expressed opinion. For example, we may modify the term "high" by roughly, more or less or definitely (which can be omitted). Formally, linguistic variables may be not original terms but terms modified by a linguistic hedge. Generally, linguistic hedges (modifiers) are special linguistic expressions by which linguistic terms are modified. They can be classified into two categories, i.e., the intensive hedges (such as very) and the weakened hedges (such as more or less). Some hedges move one term to another, while some intensify or weaken the degree of a term. It is meaningful to model this kind of linguistic expressions in MCGDM directly.

Thus we focus on the development of enabling linguistic hedges, such as more or less and roughly, in MCGDM. We shall introduce the linguistic hedges with the inclusive interpretation into the framework of MCGDM in this paper. Linguistic terms and linguistic terms modified by hedges are called linguistic expressions. Hedges are modeled by upper approximations of terms, and then an MCGDM approach is proposed to deal with this kind of evaluations where linguistic evaluations are formed by linguistic terms modified by hedges such as more or less and roughly.

\section{LiNGUISTIC TERM SETS AND FuZZY HEDGES}

Given a nonempty domain $X$, a fuzzy set $F$ in $X$ is characterized by its membership function $F: X \rightarrow[0,1]$. The class of all fuzzy sets on $X$ is denoted by $\mathcal{F}(X)$. Furthermore, for any $A, B \in \mathcal{F}(X)$, the inclusion $A \subseteq B$ stands iff $A(x) \leq B(x)(\forall x \in X)$.

The use of linguistic information is very straightforward and suitable to express the satisfaction associated with an outcome and a state of nature. Herrera et al. [7] introduced a finite and totally ordered discrete LTS described by: 


$$
S=\left\{s_{0}, s_{1}, \cdots, s_{\tau}\right\}
$$

A 3-tuple $(a, b, c)$ is called a triangular fuzzy number (TFN) if for any $x \in X$, the membership function is defined by

$$
s_{\alpha}(x)=\left\{\begin{array}{l}
(x-a) /(b-a), \max (a, 0) \leq x \leq b \\
(c-x) /(c-b), b \leq x \leq \min (c, 1) \\
0, \text { otherwise }
\end{array}\right.
$$

A simple way to compare TFNs is to distinguish their expected values [8]. The expected value of $s_{\alpha}$ is

$$
E\left(s_{\alpha}\right)=\left((1-\omega) a_{\alpha}+b_{\alpha}+\omega c_{\alpha}\right) / 2
$$

where $\omega \in[0,1]$ is an index of risk-bearing attitude of DM. For simplification, $\omega$ is usually fixed to 0.5 in application. To aggregate a set of TFNs, several operators have been proposed, such as the TFWA operator and the TFOWA operator [9]. Various tools can be adopted to obtain the weighting vector of the TFOWA operator, see [10] for detail.

Figure 1 shows a uniformly distributed semantic [7] and a non-uniformly distributed semantic [11] for a LTS with seven terms. Note that we use $s_{\alpha}$ to represent both the linguistic term and its corresponding fuzzy set.

Since the powering model introduced by Zadeh [12], linguistic hedges have been widely investigated. De Cock and Kerre [13] proposed the following general definitions of linguistic hedges by fuzzy sets:

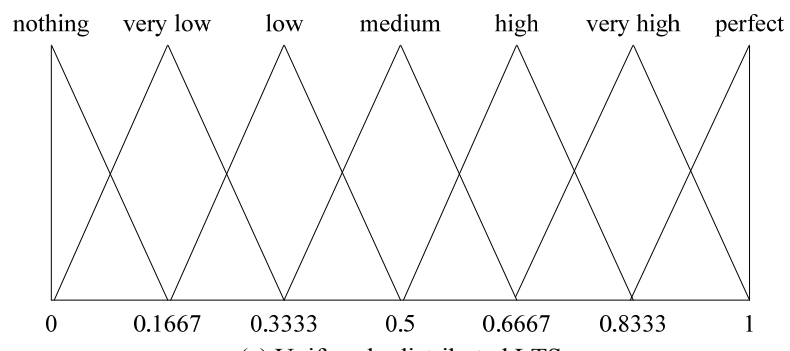

(a) Uniformly distributed LTS

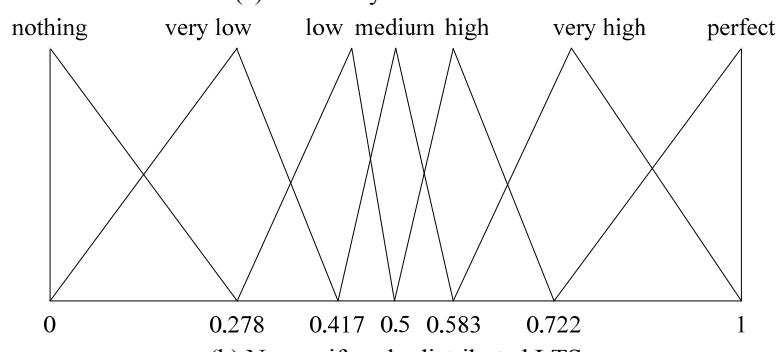

(b) Non-uniformly distributed LTS

FIGURE I. A SET OF SEVEN TERMS WITH ITS SEMANTICS.

Definition 1 [13]. Given a nonempty domain $X$, a fuzzy hedges $h$ on $X$ is a $\mathcal{F}(X) \rightarrow \mathcal{F}(X)$ mapping.

There are two interpretations of linguistic hedges, which are the inclusive interpretation and the non-inclusive interpretation [14]. The later has been widely used in linguistic MCGDM. This kind of hedge modifies a base term to different categories rather than a subset or a superset of it. The semantic entailment [15] is always assumed to be hold in the inclusive interpretation. Given $x$, the underlying semantics is that $x$ is very $A$ is also $A$ and $x$ is $A$ is also more or less $A$. That is, for $A \in \mathcal{F}(X)$ and $x \in X$,

$$
x \text { is very } A \Rightarrow x \text { is } A \Rightarrow x \text { is more or less } A
$$

Using denotations of fuzzy sets, (4) can be rewritten as:

$$
\text { very } A \subseteq A \subseteq \text { more or less } A
$$

It is clear that only weakened hedges are possible to be used in MCGDM with uncertainties. We shall focus on modeling weakened hedges, such as more or less and roughly.

\section{LINGUISTIC FuZZY ROUGH SETS}

Let $X$ be a nonempty domain, without loss of generality, we let $X=[0,1]$ in this paper. The pair $(X, R)$ (where $R$ is a fuzzy relation on $X$ ) constructs the central notion of fuzzy rough sets and is called fuzzy approximation space [16]. For $x \in X$, the fuzzy equivalence class $[x]_{R}$ is a fuzzy set $[x]_{R}(y)=R(x, y) \quad(\forall y \in X)$ indicating the degree of $y$ similar to $X$, where $R$ is a binary fuzzy relation on $X^{2}$. Dubois and Prade [17] introduced the concept of fuzzy rough sets by extending the equivalence relation of classical rough sets to fuzzy equivalence relation.

Definition 2 [17]. Given a nonempty domain $X$, a fuzzy relation $R$ and a fuzzy set $F$ on $X$. The lower and upper approximations of $F$ are defined as: $\forall y \in X$

$$
\begin{aligned}
& R \downarrow F(y)=\inf _{x \in X} \mathcal{I}(R(x, y), F(x)), \\
& R \uparrow F(y)=\sup _{x \in X} \mathcal{T}(R(x, y), F(x)),
\end{aligned}
$$

where $\mathcal{I}$ and $\mathcal{T}$ are the implication operator and the t-norm respectively.

Given $F$ on $X, R \downarrow F \subseteq F \subseteq R \uparrow F \subseteq R \uparrow \uparrow F$ holds, where $R \uparrow \uparrow F=R \uparrow(R \uparrow F)$. The pair of lower and upper approximations is called a fuzzy rough set. If $F$ is a linguistic term, we have:

Definition 3. Given a nonempty domain $X$, a fuzzy relation $R$ and a linguistic term $s_{\alpha}$ represented by fuzzy set $F$ on $X$, the pair of lower and upper approximations of $s_{\alpha}$ is called a linguistic fuzzy rough set.

We will use the upper approximation of linguistic fuzzy rough set to model linguistic hedges in the following part. 


\section{A. Resemblance Relations}

De Cock and Kerre [18] suggested a pseudometric-based method to model similarity.

Definition 4 [18]. $d$ is called a $\mathcal{S}$-pesudometric on $X$ iff for all $x, y, z \in X:(1) d(x, x)=0$, (2) $d(x, y)=d(y, x)$, and (3) $\mathcal{S}(d(x, y), d(y, z)) \geq d(x, z)$, where $\mathcal{S}$ is a t-conorm operator.

Then a new kind of resemblance relation can be defined to satisfy the assumption that the closer two objects are to each other, the more they are approximately equal.

Definition 5. For a universe $X,(\mathcal{M}, d)$ a pseudometric space and $f$ a $X \rightarrow \mathcal{M}$ mapping, a fuzzy relation $R$ on $X$ is called $a(f, d)$-resemblance relation on $X$ iff for all $x, y, z, u \in X:$ (1) $R(x, x)=1$, (2) $R(x, y)=R(y, x)$, and (3) $d(x, y) \leq d(z, u)$ implies $R(x, y) \geq R(z, u)$.

We specify the distance measure by the following form.

Definition 6. Given $x, y \in X$, the distance between $x$ and $y$ is defined by

$$
d(x, y)=|\varphi(x)-\varphi(y)|
$$

where $\varphi: X \rightarrow X$ is a monotonic increasing function.

Given linguistic terms $s_{\alpha}=\left(a_{\alpha}, b_{\alpha}, c_{\alpha}\right)(\alpha=0,1, \cdots, \tau)$ on domain $X, b_{\alpha}(\alpha=1, \cdots, \tau-1)$ can be seen as $\tau-1$ points in $X$ such that $s_{\alpha}\left(b_{\alpha}\right)=1$. To ease of representation, let $b_{-\alpha}=-\alpha b_{1}$ and $b_{\tau+\alpha}=1+\alpha\left(b_{\tau}-b_{\tau-1}\right)$. In most real cases, $s_{\alpha}$ can be denoted by $\left(b_{\alpha-1}, b_{\alpha}, b_{\alpha+1}\right)$. Let

$$
\varphi(x)=k\left(b_{\alpha}-\left(\left(b_{\alpha}-x\right) /\left(b_{\alpha}-b_{\alpha-1}\right)\right)(1 / \tau)\right)
$$

where $x \in\left[b_{\alpha-1}, b_{\alpha}\right]$ and $1 / k$ is the threshold such that the degree of $x$ is resembling to $y$ is bigger than 0. Obviously, $\varphi$ and $k$ are determined by the semantics of the LTS objectively. If the LTS is uniformly distributed, then $k$ should be $\tau$ and (7) reduces to $\varphi(x)=k x$. Then the resemblance relation can be

$$
R(x, y)=\max (0,1-d(x, y))
$$

Note that if the semantics of given LTS is not represented by TNFs, then some more complex function $\varphi$ should be defined according to the semantics.

\section{B. Representational Issues}

We will study the mathematical representation of linguistic expressions built up by the following scheme:

\section{Scheme 1}

$<$ hedge $>$ := roughly $\mid$ more or less; term $>$.

$<$ linguistic expression $>:=<$ base term $>\mid<$ hedge $><$ base

where $<$ base term $>$ is an original term of a given LTS. We focus on two weakened hedges, i.e., roughly and more or less, which may be used to represent evaluations in linguistic MCGDM problems. An intuition is that roughly has more weakening force than more or less $[15,19]$. We assume the following semantic entailment holds: for $s_{\alpha} \in S$ and $x \in X$,

$$
x \text { is } s_{\alpha} \Rightarrow x \text { is more or less } s_{\alpha} \Rightarrow x \text { is roughly } s_{\alpha}
$$

By means of fuzzy sets, (9) corresponds to:

$$
s_{\alpha} \subseteq \text { more or less } s_{\alpha} \subseteq \text { roughly } s_{\alpha}
$$

Then linguistic hedges can be modeled by means of approximations. Let $R$ be a $(f, d)$-resemblance relation on $X$, then $(X, R)$ is a fuzzy approximation space because of the reflexive and symmetric properties of $R$. Moreover, for every $F \in \mathcal{F}(X), x \in X, R \uparrow F(x)$ is the degree to which the fuzzy set of objects resembling $x$ overlaps with $F$. Then according to Definition 2 and (10), we present the following way of modeling weakened hedges: given an original term $s_{\alpha}$

$$
\begin{gathered}
\text { more or less } s_{\alpha}=R \uparrow s_{\alpha} \\
\text { roughly } s_{\alpha}=R \uparrow \uparrow s_{\alpha}
\end{gathered}
$$

According to the resemblance relation in (8), we can get the following conclusion by some easy but trial computation.

Theorem 1. Given a collection of original terms $s_{\alpha}=\left(b_{\alpha-1}, \quad b_{\alpha}, b_{\alpha+1}\right), \alpha=0,1, \cdots, \tau$, and a resemblance relation $R$ defined by (8). Let $\mathcal{T}$ be the min operation, $k=\tau$, then $\forall y \in[0,1]$

$$
\begin{gathered}
R \uparrow s_{\alpha}(y)=\left(b_{\alpha-2}, b_{\alpha}, b_{\alpha+2}\right) \\
R \uparrow \uparrow s_{\alpha}(y)=\left(b_{\alpha-3}, b_{\alpha}, b_{\alpha+3}\right)
\end{gathered}
$$

If the linguistic tem set is uniformly distributed, then (13) and (14) can be rewritten as:

$$
\begin{gathered}
R \uparrow s_{\alpha}=((\alpha-2) / \tau, \alpha / \tau,(\alpha+2) / \tau) \\
R \uparrow \uparrow s_{\alpha}=((\alpha-3) / \tau, \alpha / \tau,(\alpha+3) / \tau)
\end{gathered}
$$

Take the term "medium" from (a) of Figure 1 for example, 
"more or less medium" and "roughly medium" are shown in Figure 2.

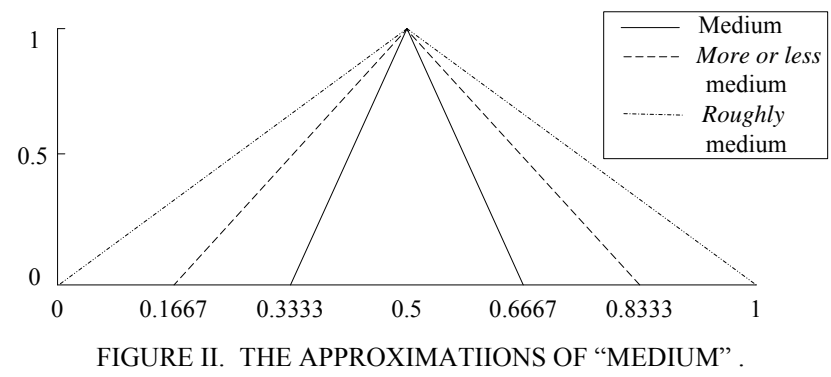

Note that the proposed approach is far form some existing approaches where some parameters are needed to be determined artificially so that the membership of a term can be modified to an acceptable level. Different form [15, 19], we concentrate on the application of fuzzy relations in MCGDM in linguistic setting. Thus we seek for specific relations to model linguistic hedges when a LTS is prepared. When the semantics of a LTS is defined by TFNs, the proposed formula can be used to compute linguistic terms with hedges according to the corresponding TFNs.

\section{MCGDM WITH LiNGUISTIC HEDGES}

We are going to present an approach for MCGDM problems in this section. Suppose that a decision organization formed by a group of DMs is authorized to evaluate $m$ alternatives with respect to a set of criteria. The organization provides the assessment information according to a predefined LTS $S$, where the assessment information of each DM can be represented by a matrix whose entries are linguistic variables having the form of both original terms and linguistic expressions with hedges. The problem is to rank alternatives based on the set of matrices. The resolution is presented step by step as follows:

Step 1. The choice of the LTS with its semantic. It is necessary to determine the granularity, labels and semantic of the LTS. Then the domain of linguistic expressions can be established to evaluate the alternatives according to the criteria. Theoretically, the resemblance relation is fixed when the semantic linguistic terms are prepared. Especially, if the LTS $S$ is uniformed distributed, the original terms are represented by triangular membership functions, and then the resemblance relation defined by (8) can be used.

Step 2. Evaluations. The decision organization is asked to evaluate alternatives with respect to each criterion and express the performance opinions by linguistic variables. The linguistic variable can be either original term $s_{\alpha} \in S$ or linguistic expression, as defined in Scheme 1, constructed by hedges (more or less and roughly) and original terms. The results of the step are a set of linguistic matrices and each of which is the performance evaluations provided by a DM.

Step 3. The choice of the aggregation operator. The DMs have to establish or select an appropriate aggregation operator, e.g., the TFOWA operator, for fusing the linguistic expressions of the DMs and then combining the fused information for each alternatives. The choice is mainly depend on the weighting vector and the preference of the decision organization. For example, if the weighting vector is unknown, or DMs only want to determine weights according to the input values, then the TFOWA operator is a good choice.

Step 4. The choice of the best alternatives. This step is usually consisted of two phases: the aggregation phase and the exploitation phase. The former combines the linguistic information provided in Step 2 by means of the aggregation operator chosen in Step 3. Then the latter establishes a priority among the alternatives and chooses the best alternative(s).

\section{APPLICATION IN EVALUATIONS OF ENERGY TECHNOLOGIES}

A sustainable energy system is crucial for any countries and the key mean is the implementation of new and innovative energy technologies [20]. The function of governments is to appraise and select energy technologies and then support the outstanding ones. However, the evaluations are really complex because of a series of uncertainties and implications that have to be encountered.

The government formed a working organization with 3 DMs, denoted by $e_{i}(i=1,2,3)$. To assess the technologies' impact on the environmental, social, economical and technological dimension of sustainable development, a number of criteria have been selected as shown in Table 1. Furthermore, the organization looked systematically into the longer-term future, sought for the technologies which have not been used in any energy sector or have been applied at the initial stage, but are likely to uphold the sustainable development by the four dimensions in Table 1. At last, four technologies were preselected as alternatives, which are $A_{1}$ : Pressurized Fluidized Bed Combustion, $A_{2}$ : Natural Gas Combined Cycle, $A_{3}$ : Fuel Cell/Turbine Hybrids and $A_{4}$ : Biomass Gasification. We solve the problem by the approach proposed in Section IV as follows.

TABLE I. SELECTED CRITERIA OF APPRAISING ENERGY TECHNOLOGIES .

\begin{tabular}{|l|l|}
\hline Dimension & Criterion \\
\hline \multirow{3}{*}{ Economic } & $c_{1}:$ Investment cost \\
\cline { 2 - 2 } & $c_{2}:$ Economic viability using payback period \\
\hline \multirow{4}{*}{ Environmental } & $\begin{array}{l}c_{3}: \text { Contribution to confrontation of the climate change } \\
\text { phenomenon }\end{array}$ \\
\cline { 2 - 2 } & $c_{4}:$ Effects on natural environment \\
\hline \multirow{3}{*}{ Technological } & $c_{5}:$ Efficiency rate \\
\cline { 2 - 2 } & $c_{6}:$ Knowledge of the innovative technology \\
\hline \multirow{3}{*}{ Social } & $c_{7}:$ Contribution to employment opportunities' creation \\
\cline { 2 - 2 } & $c_{8}:$ Contribution to regional development \\
\hline
\end{tabular}

Step 1. Considering the recommendation of the decision organization, the LTS $S$ in Fig. 1(a) is used. Thus $\tau=6$ and $s_{\alpha}=\left(a_{\alpha}, b_{\alpha}, c_{\alpha}\right)=(\alpha-1 / \tau, \alpha / \tau, \alpha+1 / \tau)$ for $\alpha=1,2, \cdots, \tau$. The resemblance relation is defined by (8). 
Step 2. Two weakened hedges (more or less and roughly) can be used to represent uncertain and hesitant opinions when evaluating. Based on the domain of linguistic expressions, the performances of technologies provided by $3 \mathrm{DMs}$ are presented in Table 2.

Step 3. The weights of 3 DMs are 0.3, 0.3 and 0.4. Thus we use the THWA operator to aggregate 3 matrices shown in Table 2. Moreover, the organization insists that the importance of 8 criteria is equal. In this case the TFOWA operator can be used to fuse the resultant matrix of TFNs for each alternative. Yager [21] suggested an interesting way to derive the associated weighting vector of the TFOWA operator by means of a fuzzy linguistic quantifier. The weighting vector for the fuzzy quantifiers "As many as possible" can be defined as $W=(0,0,0,0,0.25,0.25,0.25,0.25)^{T}[20]$.

Step 4. The aggregation result of 4 alternatives is shown in Figure 3. Using the expected value defined in (3), the ranking of alternatives is: $A_{2} \succ A_{1} \succ A_{4} \succ A_{3}$. Thus the Natural Gas Combined Cycle is the most desirable technology. However, the Fuel Cell/Turbine Hybrids cannot be considered.

TABLE II. PERFORMANCES OF TECHNOLOGIES PER CRITERION .

\begin{tabular}{|l|l|l|l|l|l|l|l|l|l|}
\hline DM & $\begin{array}{l}\text { Altern } \\
\text { ative }\end{array}$ & $c_{1}$ & $c_{2}$ & $c_{3}$ & $c_{4}$ & $c_{5}$ & $c_{6}$ & $c_{7}$ & $c_{8}$ \\
\hline \multirow{5}{*}{$c_{1}$} & $A_{1}$ & $\mathrm{VH}$ & $\mathrm{H}$ & $\mathrm{VH}$ & $m \mathrm{VH}$ & $\mathrm{H}$ & $\mathrm{H}$ & $r \mathrm{H}$ & $\mathrm{H}$ \\
\cline { 2 - 11 } & $A_{2}$ & $\mathrm{VH}$ & $\mathrm{H}$ & $\mathrm{VH}$ & $\mathrm{VH}$ & $\mathrm{VH}$ & $m \mathrm{P}$ & $\mathrm{VH}$ & $\mathrm{H}$ \\
\cline { 2 - 10 } & $A_{3}$ & $m \mathrm{~N}$ & $\mathrm{M}$ & $\mathrm{H}$ & $\mathrm{VH}$ & $\mathrm{M}$ & $\mathrm{VL}$ & $\mathrm{L}$ & $\mathrm{L}$ \\
\cline { 2 - 11 } & $A_{4}$ & $\mathrm{M}$ & $\mathrm{H}$ & $\mathrm{L}$ & $\mathrm{H}$ & $m \mathrm{VH}$ & $\mathrm{H}$ & $\mathrm{P}$ & $\mathrm{VH}$ \\
\hline \multirow{5}{*}{$c_{2}$} & $A_{1}$ & $\mathrm{H}$ & $m \mathrm{H}$ & $\mathrm{VH}$ & $\mathrm{VH}$ & $r \mathrm{H}$ & $\mathrm{H}$ & $\mathrm{M}$ & $m \mathrm{H}$ \\
\cline { 2 - 10 } & $A_{2}$ & $\mathrm{VH}$ & $\mathrm{M}$ & $\mathrm{H}$ & $m \mathrm{P}$ & $\mathrm{VH}$ & $\mathrm{VH}$ & $\mathrm{VH}$ & $\mathrm{M}$ \\
\cline { 2 - 10 } & $A_{3}$ & $\mathrm{~L}$ & $\mathrm{~L}$ & $m \mathrm{H}$ & $\mathrm{H}$ & $r \mathrm{H}$ & $\mathrm{L}$ & $m \mathrm{~L}$ & $\mathrm{VL}$ \\
\cline { 2 - 10 } & $A_{4}$ & $\mathrm{H}$ & $\mathrm{M}$ & $m \mathrm{M}$ & $\mathrm{H}$ & $\mathrm{VH}$ & $r \mathrm{H}$ & $\mathrm{H}$ & $\mathrm{H}$ \\
\hline \multirow{5}{*}{$c_{3}$} & $A_{1}$ & $r \mathrm{H}$ & $\mathrm{VH}$ & $\mathrm{H}$ & $m \mathrm{H}$ & $\mathrm{H}$ & $\mathrm{H}$ & $m \mathrm{H}$ & $\mathrm{VH}$ \\
\cline { 2 - 10 } & $A_{2}$ & $\mathrm{H}$ & $\mathrm{H}$ & $m \mathrm{H}$ & $\mathrm{H}$ & $\mathrm{M}$ & $\mathrm{VH}$ & $\mathrm{H}$ & $\mathrm{VH}$ \\
\cline { 2 - 10 } & $A_{3}$ & $\mathrm{VL}$ & $\mathrm{H}$ & $m \mathrm{H}$ & $m \mathrm{VH}$ & $\mathrm{M}$ & $\mathrm{VL}$ & $\mathrm{M}$ & $\mathrm{L}$ \\
\cline { 2 - 10 } & $A_{4}$ & $\mathrm{M}$ & $m \mathrm{H}$ & $\mathrm{M}$ & $\mathrm{H}$ & $\mathrm{H}$ & $\mathrm{H}$ & $\mathrm{VH}$ & $m \mathrm{VH}$ \\
\hline
\end{tabular}

$\mathrm{N}=$ Nothing, $\mathrm{VL}=$ Very Low, $\mathrm{L}=$ Low, $\mathrm{M}=$ Medium, $\mathrm{H}=$ High, $\mathrm{VH}=$ Very High, $\mathrm{P}=\mathrm{Perfect}, \mathrm{m}=$

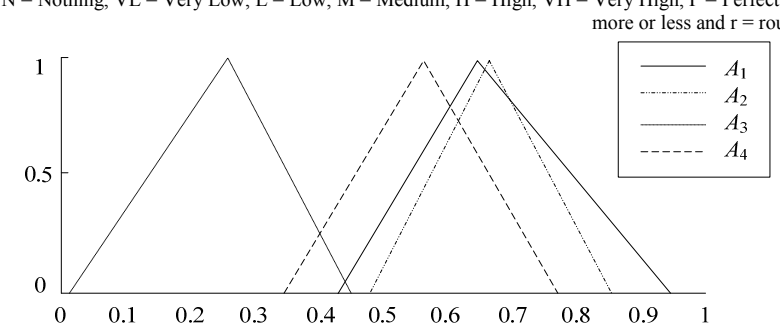

FIGURE III. COMPUTATIONAL RESULTS OF ALTERNATIVES.

\section{CONCLUSIONS}

Linguistic hedges are rational and direct tools for experts to express qualitative evaluations under uncertainties. But hedges with non-inclusive interpretation had not been investigated in
MCGDM. Based on the perspective of fuzzy sets, we have modeled hedges by means of approximations of the original terms. A novel resemblance relation has been developed base on the pseudometric space to model approximate equality of two objects in the domain. Then the upper approximation can be obtained based on the definition of fuzzy rough set. Then the linguistic fuzzy rough set is determined and an approach is presented for MCGDM problems whose evaluation information includes linguistic hedges.

\section{REFERENCES}

[1] J. Ma, J. Lu, and G. Zhang, "Decider: A fuzzy multi-criteria group decision support system," Knowl-based Syst, vol. 23, pp. 23-31, 2010.

[2] L. A. Zadeh, "The concept of a linguistic variable and its application to approximate reasoning-III," Inform Sciences, vol. 9, pp. 43-80, 1975.

[3] R. M. Rodriguez and L. Martinez, "An analysis of symbolic linguistic computing models in decision making," Int J Gen Syst, vol. 42, pp. 121136, 2013.

[4] L. A. Zadeh, "The concept of a linguistic variable and its application to approximate reasoning--I," Inform Sciences, vol. 8, pp. 199-249, 1975.

[5] M. Delgaodi, M. Vila, and W. Voxman, "On a canonical representation of fuzzy numbers," Fuzzy Set Syst, vol. 93, pp. 125-135, 1999.

[6] R. M. Rodriguez, L. Martinez, and F. Herrera, "Hesitant Fuzzy Linguistic Term Sets for Decision Making," Ieee T Fuzzy Syst, vol. 20, pp. 109-119, 2012.

[7] F. Herrera, E. Herrera-Viedma, and L. Martínez, "A fusion approach for managing multi-granularity linguistic term sets in decision making," Fuzzy Set Syst, vol. 114, pp. 43-58, 2000.

[8] T.-S. Liou and M.-J. J. Wang, "Ranking fuzzy numbers with integral value," Fuzzy Set Syst, vol. 50, pp. 247-255, 1992.

[9] Z. S. Xu, "A fuzzy ordered weighted geometric operator and its application to in fuzzy AHP," J Syst Eng Electron, vol. 31, pp. 855-858, 2002.

[10] Z. S. Xu, "An overview of methods for determining OWA weights," Int J Intell Syst, vol. 20, pp. 843-865, Aug 2005.

[11] Z. S. Xu, "An Interactive Approach to Multiple Attribute Group Decision Making with Multigranular Uncertain Linguistic Information," Group Decis Negot, vol. 18, pp. 119-145, Mar 2009.

[12] L. A. Zadeh, "A fuzzy-set-theoretic interpretation of linguistic hedges," Journal of Cybernetics, vol. 2, pp. 4-34, 1972.

[13] M. De Cock and E. E. Kerre, "Fuzzy modifiers based on fuzzy relations," Inform Sciences, vol. 160, pp. 173-199, 2004.

[14] E. E. Kerre and M. De Cock, "Linguistic modifiers: an overview," in Fuzzy Logic and Soft Computing. vol. 9, G. Chen, M. Ying, and K. Y. Cai, Eds., ed Norwell: Kluwer Academic Publishers, 1999, pp. 69-85.

[15] G. Lakoff, "Hedges: a study in meaning criteria and the logic of fuzzy concepts," Journal of Philosophical Logic, vol. 2, pp. 458-508, 1973.

[16] A. M. Radzikowska and E. E. Kerre, "A comparative study of fuzzy rough sets," Fuzzy Set Syst, vol. 126, pp. 137-155, 2002.

[17] D. Dubois and H. Prade, "Rough fuzzy sets and fuzzy rough sets," Int $J$ Gen Syst, vol. 17, pp. 191-209, 1990.

[18] M. De Cock and E. Kerre, "On (un)suitable fuzzy relations to model approximate equality," Fuzzy Set Syst, vol. 133, pp. 137-153, 2003.

[19] M. De Cock, A. M. Radzikowska, and E. E. Kerre, "A fuzzy-rough approach to the representation of linguistic hedges," in Technologies for Constructing Intelligent Systems 1: Tasks. vol. 89, B. BouchonMeunier, J. GutierrezRios, L. Magdalena, and R. R. Yager, Eds., ed, 2002, pp. 33-42.

[20] H. C. Doukas, B. M. Andreas, and J. E. Psarras, "Multi-criteria decision aid for the formulation of sustainable technological energy priorities using linguistic variables," Eur J Oper Res, vol. 182, pp. 844-855, 2007.

[21] R. R. Yager, "On ordered weighted averaging aggregation operators in multi-criteria decision making," IEEE T Syst Man Cyb, vol. 18, pp. 183$190,1988$. 